American J. of Engineering and Applied Sciences 3 (2): 260-264, 2010

ISSN 1941-7020

(C) 2010 Science Publications

\title{
The Optical Nonlinearity of Au and Ag Nanoparticle Prepared by the $\boldsymbol{\gamma}$-Radiation Method
}

\author{
Esmaeil Shahriari, W. Mahmood Mat Yunus, Kazem Naghavi and Elias Saion \\ Department of Physics, Faculty of Science, \\ University Putra Malaysia, 43400 UPM, Serdang, Malaysia
}

\begin{abstract}
Problem statement: The third order nonlinear optical properties of metal nanoparticles have been of interest in physical chemistry, medical diagnostics and optical devices. Gold colloidal nanoparticles are responsible for the brilliant reds seen in stained glass windows and silver particles are typically yellow. The purpose of the study was to determine the nonlinear refraction and absorption coefficient of the Au and Ag nanoparticles in PVP solution. Approach: The samples were prepared by $\gamma$-radiation method and the nonlinear optical properties of the composites were investigated using a single beam Z-scan technique with a beam power of $40 \mathrm{~mW}$ and operated at wavelength of $532 \mathrm{~nm}$. The measurements were carried out for both Open and closed aperture Z-scan arrangements. Results: For both Au/PVP and Ag/PVP samples the results exhibited reverse saturable absorption. The closed aperture Z-scan of the nano-fluid samples revealed self-defocusing effect while the open aperture Z-scan of the samples show a reversible saturable absorption. Conclusion: The Z-scan measurement showed that silver and gold nano-fluid prepared by gamma radiation exhibited large thermal nonlinear refractive index $\mathrm{n}_{2}$ as $-8.78 \times 10^{-7}$ and $-2.478 \times 10^{-6} \mathrm{~cm}^{2} / \mathrm{W}$, respectively. We have also investigated nonlinear absorption of these samples and we found a large value of nonlinear absorption for $\mathrm{Ag}$ nanoparticle and a weak absorption for $\mathrm{Au}$ nanoparticle. In conclusion, the experimental result shows a good nonlinear refractive index at low laser power in which encouraging for possible applications in nonlinear optical devices.
\end{abstract}

Key words: Z-scan, metal nanoparticles, optical properties

\section{INTRODUCTION}

Colloidal solutions containing metal nanoparticles can be prepared by different methods, such as laser ablation method (Ganeev et al., 2004) and chemical reaction method (Henglein and Giersig, 1999; Lee and Meisel, 1982). Lately, in situ synthesis technique has been developed to prepare noble metal colloidal solution with well-dispersion and high concentration (Karthikeyan et al., 2006). There are many literatures reporting the nonlinear optical response of metal colloids, but nonlinear optical parameters of colloidal metals vary at various stages of aggregation. Because the aggregation of colloidal metals can be excluded in the solid state, therefore it will be more valuable to investigate the optical nonlinearity of metal nanoparticles.

The z-scan measurement technique (Sheik-Bahae et al., 1989; 1990) is often used for measuring the strength of the Kerr nonlinearity (i.e., the magnitude of the nonlinear index $\mathrm{n}_{2}$ ) and thermal nonlinearity effect of an optical material. Essentially, a sample of the material under investigation is moved through the focus of a laser beam and the beam radius (or the on-axis intensity) is measured at some point behind the focus as a function of the sample position. These quantities are affected by the self-focusing effect. If the nonlinear refractive index is positive and the sample is placed behind the focus, self-focusing reduces the beam divergence and thus increases the detector signal. If the sample is moved to the left-hand side of the focus and the stronger divergence after the focus decreases the detector signal. From the measured dependence of the detector signal on the sample position, it is possible to calculate the magnitude of the nonlinear index. Nonlinear absorption due to two-photon absorption, can also affect the measured signal. This, however, can be measured separately by recording the power of the whole transmitted beam. With these data, the measurement of nonlinearity can be corrected.

In present work, we presented our studies of nonlinear optical properties of Au and Ag nanoparticles

Corresponding Author: W. Mahmood Mat Yunus, Department of Physics, Faculty of Science, University Putra Malaysia, 43400 UPM, Serdang, Malaysia Tel :+( 603)89466684 
doped in Polyvinylpyrrolidone (PVP) solution, in which $\mathrm{Au}$ and Ag nanoparticles were prepared by $\gamma$-radiation method. We investigated the nonlinear refraction and nonlinear absorption using $\mathrm{CW}$ laser beam excitation of $532 \mathrm{~nm}$.

\section{MATERIALS AND METHODS}

Silver nitrate, $\mathrm{AgNO}_{3} \quad$ (Aldrish-99\%), polyvinylpyrrolidone, PVP (MW 29,000 Aldrish), Citrate trisodium (Fluke 95\%) isopropanol were used for preparing Ag nano-fluid sample. The PVP and isopropanol were used as a colloidal stabilizer and radical scavenger of hydroxyl radical respectively. The PVP solutions were made by dissolving PVP powder in deionized water at room temperature. The solution was magnetically stirred for $2 \mathrm{~h}$ and was bubbled with nitrogen gas $(99.5 \%)$ in order to remove oxygen.

$\gamma$-Radiation $\left({ }^{60}\right.$ Co-rays) was used as an effective tool for polymerization process and reducing agent. Silver nitrate (AgNO3) at concentration of $4.709 \times 10^{-3} \mathrm{M}$ was added into $4 \mathrm{mg}$ PVP solution and isopropanol. The mixture was then irradiated with $\gamma$ radiation at doses of $30 \mathrm{kGy}$. In this process, $\gamma$ irradiation produces hydrated electrons that reduce the silver ions to silver atoms, which then aggregated and suspended in the solution. A similar procedure was applied for preparing $\mathrm{Au}$ nano-fluid sample. In this case $\mathrm{HAuCl}_{4} .3 \mathrm{H}_{2} \mathrm{O}$ at concentration of $5.886 \times 10^{-4} \mathrm{M}$ and $2.5 \mathrm{~g}$ PVP was used. The average diameters of $\mathrm{Ag}$ and $\mathrm{Au}$ nanoparticles were measured using nanophox machine (Sympatec GmbH, D-38678) and the particles size were recorded as 33.5 and $36.2 \mathrm{~nm}$, respectively.

Figure 1 shows the schematic diagram of a single beam Z-scan experiment used in the present measurement for closed aperture. The open aperture experimental set up has been previously discussed (Shahriari and Yunus, 2010). The experiments were performed using a $532 \mathrm{~nm}$ laser beam from Laserdiode (Coherent Compass SDL-532-150T). The beam was focused to a small spot using a lens and the sample was moved along a $\mathrm{z}$-axis by a motorized translational stage. The transmitted light in the far field passed through the aperture and the beam intensity was recorded by a detector $\mathrm{D}$. The laser beam waist $\omega_{0}$ at the focus length was measured to be $24.4 \mu \mathrm{m}$ and the Raleigh length was found to satisfy the basic criteria of the z-scan experiment. The linear absorption spectra for both samples were measured using UV-Vis spectrophotometer (ShimadzuUV1650PC).

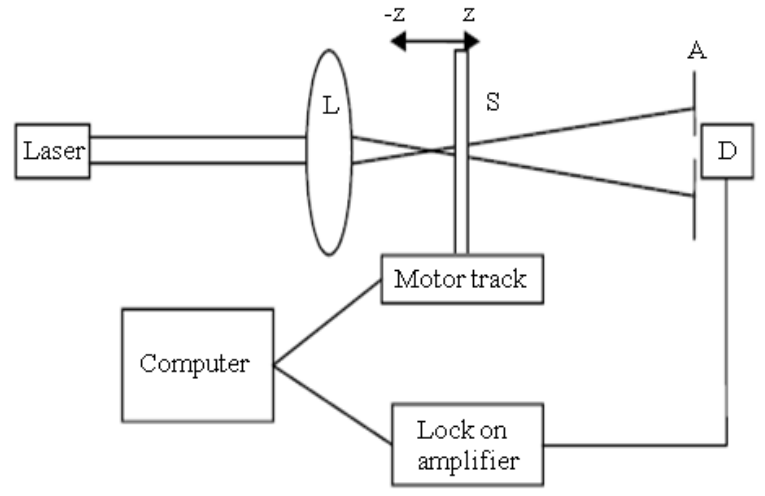

Fig. 1: Schematic diagram of a single beam Z-scan experiment setup: (L) Lens; (S) Sample; (A) Aperture; (D) Detector

\section{RESULTS}

Figure 2 and 3 show the linear absorption spectra for Ag and Au nano-fluid. The peaks show the surface plasmon resonance absorptions which are located at 520 and $410 \mathrm{~nm}$, respectively.

Figure 4 and 5 show the typical transmittance curves obtained for $\mathrm{Au}$ and $\mathrm{Ag}$ nano-fluids prepared by $\gamma$-radiation from ${ }^{60} \mathrm{Co}$ source. The laser intensity was measured to be $\mathrm{I}_{0}=4.27 \times 10^{3} \mathrm{~W} / \mathrm{cm}^{2}$ and the aperture size was 0.2 . These peak-valley curves indicate that the nonlinear refractive index of the medium is negative. The third order nonlinear refractive index, $\mathrm{n}_{2}$ was calculated using a similar method to the one as reported by Sheik-Bahae et al. (1990) and Wang et al. (1994) i.e.:

$$
\mathrm{n}_{2}=\frac{\Delta \phi_{\mathrm{o}}}{\mathrm{kL}_{\mathrm{eff}} \mathrm{I}_{\mathrm{o}}}
$$

Where:

$\mathrm{k}=2 \pi / \lambda$ is the wave factor

$\mathrm{I}_{0}=$ The beam intensity

$L_{\text {eff }}=(1-\exp (-\alpha \mathrm{L})) / \alpha$ is the effective thickness of the sample

$\alpha_{0}=$ The linear absorption of the medium

$\mathrm{L}=$ The thickness of the sample

The linear absorption coefficient $\alpha$ was obtained from the absorption spectra Fig. 2 and 3 . The $\Delta \phi_{\mathrm{o}}$ was calculated from the experimental data of the normalized peak to valley transmittance, $\Delta \mathrm{T}_{\mathrm{p}-\mathrm{v}}$ given as:

$\Delta \mathrm{T}_{\mathrm{p}-\mathrm{v}} \approx 0.406(1-\mathrm{s})^{0.25}\left|\Delta \phi_{\mathrm{o}}\right|$ 
where, $\mathrm{s}$ is the aperture linear transmittance and taken to be 0.2 for the present experiment. The solid line in Fig. 4 is the calculated value using analytical equation proposed by Liao et al. (1997); (1998):

$\mathrm{T}(\mathrm{z}, \Delta \phi)=1-\frac{4 \Delta \phi_{0} \mathrm{x}}{\left(\mathrm{x}^{2}+1\right)\left(\mathrm{x}^{2}+9\right)}$

where, $\mathrm{x}=\mathrm{z} / \mathrm{z}_{0}$.

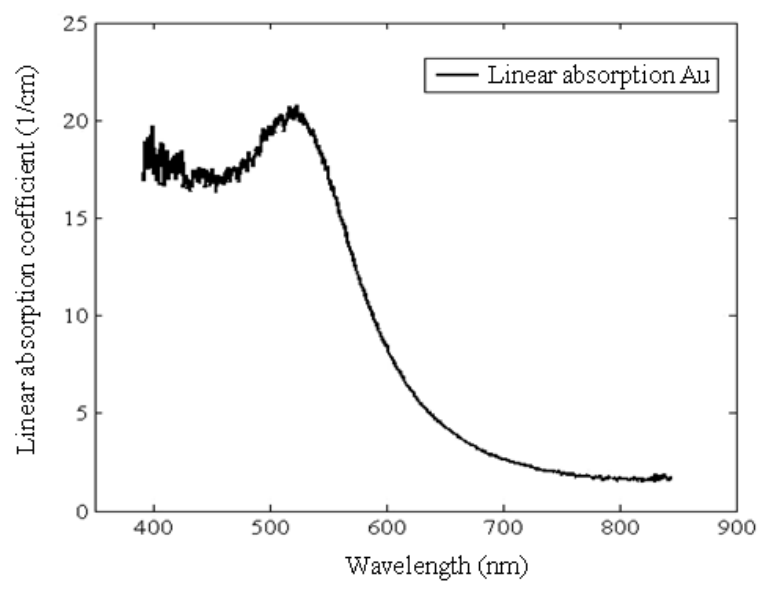

Fig. 2: Linear absorption coefficient Au/PVP nanofluid at concentration of $5.886 \times 10^{-4} \mathrm{M}$. The average particle size is $36.2 \mathrm{~nm}$

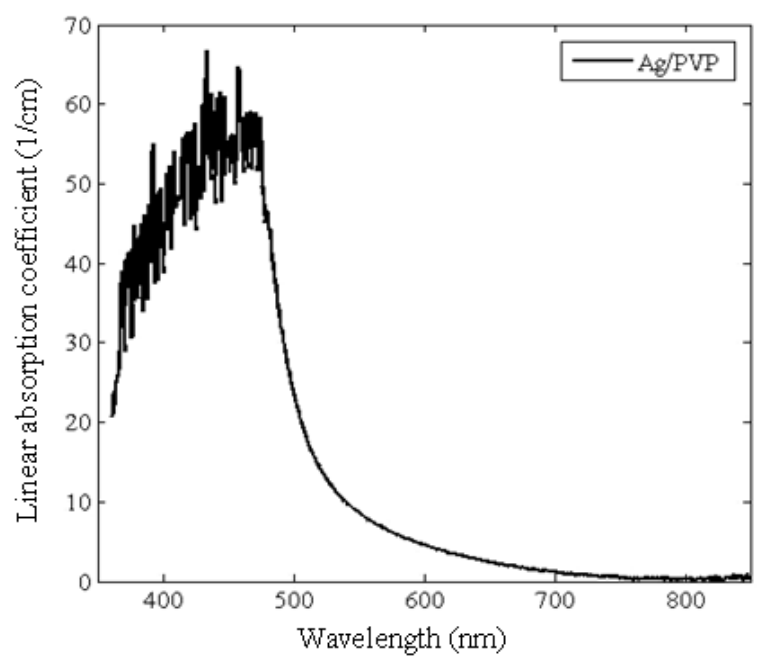

Fig. 3: Linear absorption coefficient Ag/PVP nanofluid at concentration of $4.709 \times 10^{-3} \mathrm{M}$. The average particle size is $33.5 \mathrm{~nm}$
The nonlinear absorption was obtained by analyzing the experimental data using a well known nonlinear absorption equation proposed by Sheik-Bahae et al. (1990):

$\mathrm{T}(\mathrm{z})=\sum_{\mathrm{m}=0}^{\infty}\left(\frac{\beta \mathrm{I}_{0} \mathrm{l}_{\mathrm{eff}}}{1+\mathrm{z}^{2} / \mathrm{z}_{0}^{2}}\right)^{\mathrm{m}}(\mathrm{m}+1)^{-3 / 2}$

Where:

$\mathrm{z}_{0}=\mathrm{k} \omega_{0}^{2} / 2$ is the diffraction length of the beam

$\omega_{0}=$ the beam waist radius at the focal point

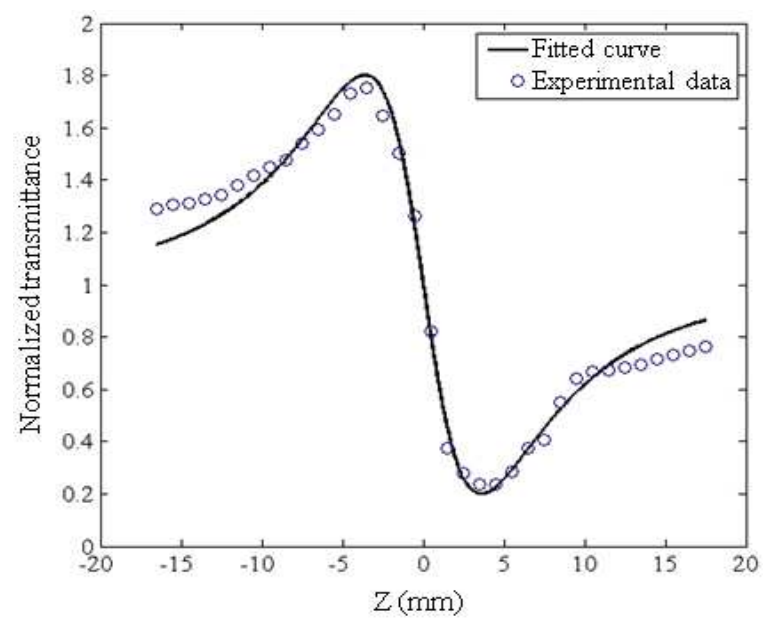

Fig. 4: Closed aperture experimental data of Au nanofluid measured at concentration of $5.886 \times 10^{-4}$ M. The average particle size is $36.2 \mathrm{~nm}$. The solid line is the theoretical curve calculated using Eq. 3

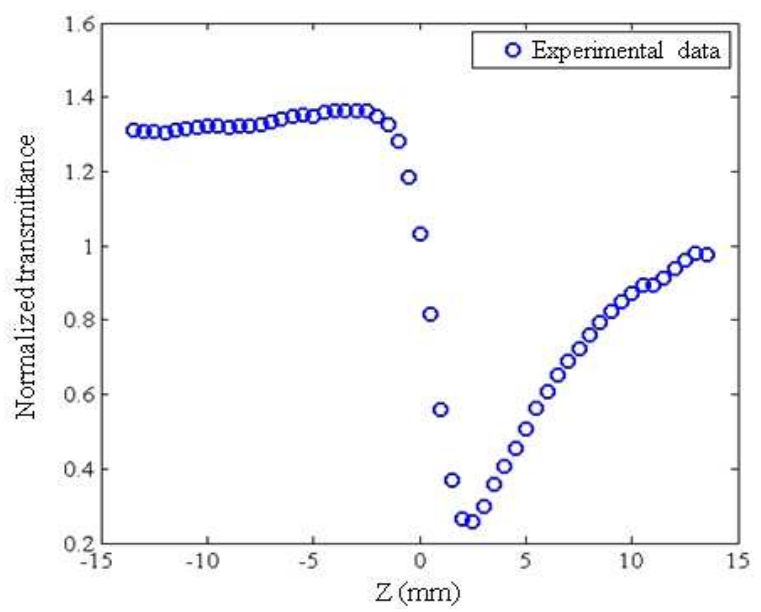

Fig. 5: Closed aperture experimental data of Ag nanofluid measured at concentration of $4.709 \times 10^{-3}$ M. The average particle size is $33.5 \mathrm{~nm}$ 
Am. J. Engg. \& Applied Sci., 3 (2): 260-264, 2010

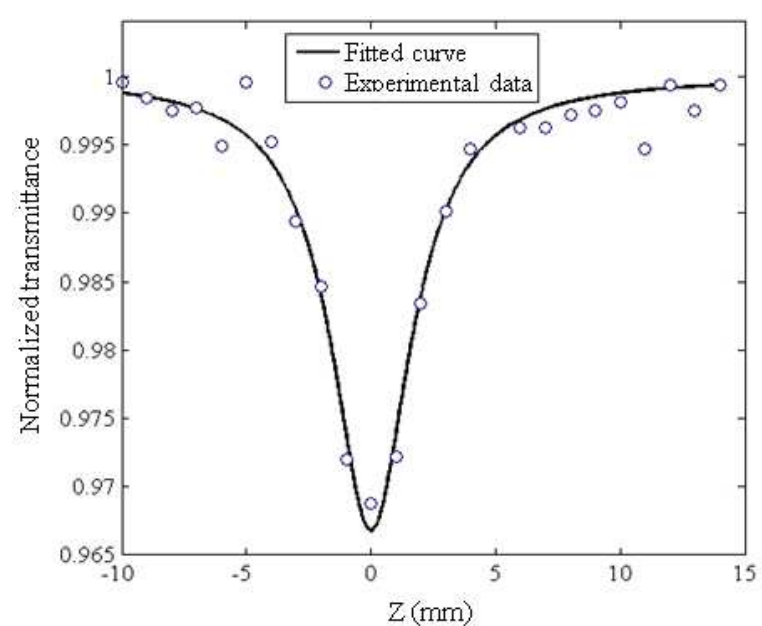

Fig. 6: Open aperture Z-scan curve for Au nanoparticle measured at a concentration of $5.886 \times 10^{-4} \mathrm{M}$ Solid line is the fitted curve using Eq. 4

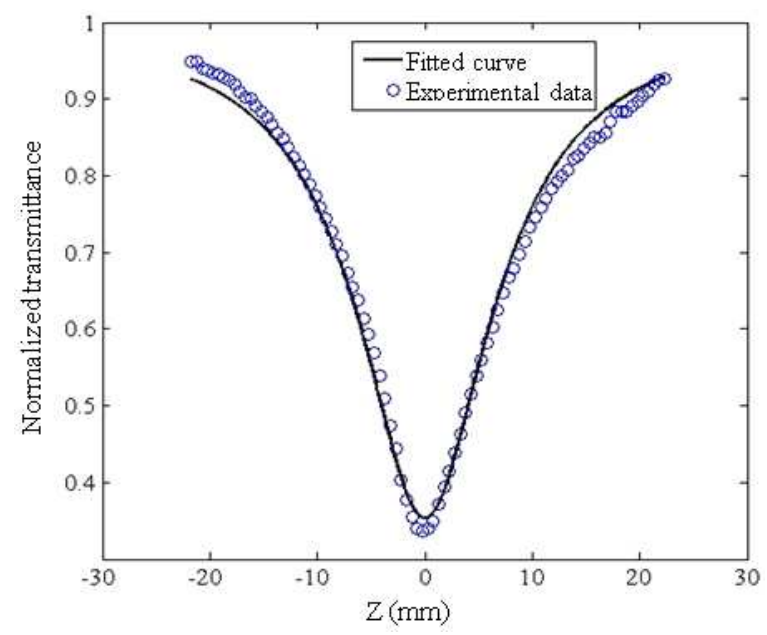

Fig. 7: Open aperture Z-scan curve for Ag nanoparticle measured at a concentration of $4.709 \times 10^{-3} \mathrm{M}$ Solid line is the fitted curve using Eq. 4

using the value of $\mathrm{I}_{0}$ and $\mathrm{s}$ measured in the present work we obtained the value of non-linear refractive index $n_{2}$ for $\mathrm{Au}$ and $\mathrm{Ag}$ nano-fluid sample as-2.47 $\times 10^{-6}$ and $-8.78 \times 10^{-7} \mathrm{~cm}^{2} / \mathrm{W}$, respectively.

Figure 6 shows the open aperture z-scan experimental data of $\mathrm{Au}$ nanoparticle while Fig. 7 displays the experimental data for $\mathrm{Ag}$ nanoparticles. The solid lines in the Fig. 6 and 7 are theoretical fits based on nonlinear absorption phenomenon described by Eq. 4. Using the laser beam intensity at the focus point was as $4.27 \times 10^{3} \mathrm{~W} / \mathrm{cm}^{2}$ we calculated the nonlinear absorption coefficient for these two types of samples. The values are listed in Table 1.
Table 1: Nonlinear optical properties of $\mathrm{Au}$ and $\mathrm{Ag}$ nano-fluid measured at $532 \mathrm{~nm}$ laser beam

\begin{tabular}{lllll}
\hline Samples & Particle size $(\mathrm{nm})$ & $\mathrm{n}_{2}\left(\mathrm{~cm}^{2} / \mathrm{W}\right)$ & $\beta(\mathrm{cm} / \mathrm{W})$ & $\alpha(\mathrm{cm}-1)$ \\
\hline $\mathrm{Au} / \mathrm{PVP}$ & 36.2 & $-2.478 \times 10^{-6}$ & $1.71 \times 10^{-3}$ & 19.110 \\
$\mathrm{Ag} / \mathrm{PVP}$ & 33.5 & $-8.780 \times 10^{-7}$ & $1.88 \times 10^{-1}$ & 11.852 \\
\hline
\end{tabular}

\section{DISCUSSION}

Whenever the sample has a nonlinear refractive index and high nonlinear absorption properties, the normalized transmission curve of closed aperture data does not show a perfect symmetry curve. This phenomenon can be clearly seen in Fig. 5 where the closed aperture data (open circle) shows a suppressed peak and enhanced valley. In Fig. 4, the suppressed peak and enhanced valley did not exist due to small nonlinear absorption coefficient of $\mathrm{Au}$ nano-fluid sample. For case in Fig. 5, the nonlinear refraction coefficient of the sample was obtained by dividing the Closed Aperture (CA) experimental data (Fig. 5) to the Open Aperture (OA) experimental data of Fig. 7. Thus $\Delta \mathrm{T}_{\mathrm{p}-\mathrm{v}}$ measured from CA/OA fitted curve (not shown) was used to calculate the third order nonlinear refractive index of Ag nanofluids as listed in Table 1.

This result shows that the Ag nano-fluid with the particle size of $33.5 \mathrm{~nm}$ gives large values of nonlinear refractive index and nonlinear absorption while the $\mathrm{Au}$ nano-fluid with the particle size of $36.2 \mathrm{~nm}$ gives a large value of nonlinear refraction coefficient and weak nonlinear absorption coefficient.

\section{CONCLUSION}

The optical thermal nonlinear coefficient refractive index of $\mathrm{Au}$ and $\mathrm{Ag}$ solution were investigated by a single beam Z-scan technique. The Z-scan measurement shown that gold and silver nanofluid prepared by gamma radiation exhibited large thermal nonlinear refractive index $\mathrm{n}_{2}$ as $-2.478 \times 10^{-6}$ and $-8.78 \times 10^{-7} \mathrm{~cm}^{2} / \mathrm{W}$, respectively. The experimental data also confirmed the nonlinearity phenomenon was due self-defocusing process. We have also investigated nonlinear absorption of these materials and found a large value for Ag nanoparticle while a weak absorption for Au nanoparticle. The nonlinear behavior of these nanoparticles at low laser power made them good candidates for possible applications in nonlinear optical devices.

\section{ACKNOWLEDGEMENT}

We gratefully acknowledge the financial support from MOSTI through Fundamental research grant (0111-08-664FR/5523664). 
Am. J. Engg. \& Applied Sci., 3 (2): 260-264, 2010

\section{REFERENCES}

Ganeev, R.A., M. Baba, A.I. Ryasnyansky, M. Suzuki and H. Kuroda, 2004. Characterization of optical and nonlinear optical properties of silver nanoparticles prepared by laser ablation in various liquids. Opt. Commun., 240: 437-448. DOI: 10.1016/j.optcom.2004.06.049

Henglein, A. and M. Giersig, 1999. Formation of colloidal silver nanoparticles: Capping action of citrate. J. Phys. Chem. B., 103: 9533-9539. DOI: 10.1021/jp9925334

Karthikeyan, B., M. Anija and M.R. Philip, 2006. In situ synthesis and nonlinear optical properties of $\mathrm{Au}: \mathrm{Ag}$ nanocomposite polymer films. Applied Phys. Lett. 88: 1-3. DOI: 10.1063/1.2168667

Lee, P.C. and D. Meisel, 1982. Adsorption and surfaceenhanced Raman of dyes on silver and gold sols. J. Phys. Chem. 86: 3391-3395. DOI: $10.1021 / \mathrm{j} 100214 \mathrm{a} 025$

Liao, H.B., R.F. Xiao, J.S. Fu, H. Wang, K.S. Wong and G.K.L. Wong, 1998. Origin of third-order optical nonlinearity in $\mathrm{Au}: \mathrm{SiO} 2$ composite films on femtosecond and picosecond time scales. Opt. Lett., 23: 388-390. DOI: 10.1364/OL.23.000388

Liao, H.B., R.F. Xiao, J.S. Fu, H. Wang, K.S. Wong, G.K.L. Wong and P. Sheng, 1997. Large third order optical nonlinearity in $\mathrm{Au}: \mathrm{SiO}_{2}$ composite films near the percolation threshold. Applied Phys. Lett., 70: 1-3.
Shahriari, E. and W.M. Yunus, 2010. Single beam Zscan measurements of nonlinear refraction and nonlinear absorption coefficients in silver nanofluid. Am. J. Applied Sci., 3: 98-101. http://www.scipub.org/fulltext/ajeas3198-101.pdf

Sheik-Bahae, M., A.A. Said and E.W. Van Stryland, 1989. High sensitivity single beam $\mathrm{n}_{2}$ measurements. Opt. Lett., 14: 955-957. DOI: 10.1364/OL.14.000955.

Sheik-Bahae, M., A.A. Said, T. Wei, D.J. Hagan and E.W. Van Stryland, 1990. Sensitive measurement of optical nonlinearities using a single beam. IEEE J. Quant. Elect., 26: 760-769. DOI: $10.1109 / 3.53394$

Wang, J., M. Sheik-Bahae, A.A. Said, D.J. Hagan and E.W. Van Stryland, 1994. Time-resolved Z-scan measurements of optical nonlinearities. J. Opt. Soc. Am. B., 11: 1009-1017. DOI: 10.1364/JOSAB.11.001009 